\title{
Transdermal 4-Hydroxytestosterone
}

National Cancer Institute

\section{Source}

National Cancer Institute. Transdermal 4-Hydroxytestosterone. NCI Thesaurus. Code C114103.

A transdermal formulation containing 4-hydroxytestosterone (4-OHT), a steroidal aromatase inhibitor (AI) and androgen receptor (AR) antagonist, with potential antineoplastic activity. 4-OHT is largely converted into 4-hydroxyandrostenedione (4$\mathrm{OHA}$ ) and irreversibly binds to and inhibits aromatase, thereby blocking the conversion of androstenedione to estrone, and testosterone to estradiol. This may inhibit tumor cell proliferation in estrogen-dependent tumor cells. In addition, 4-OHT binds to the AR and may inhibit AR-mediated tumor cell growth. Aromatase, a cytochrome P-450 enzyme, is overexpressed in a variety of cancer cells; it plays a key role in estrogen biosynthesis. Compared to oral 4-OHT, the transdermal formulation allows for continuous release of 4-OHT into the bloodstream and prevents first pass metabolism by the liver. 